\title{
Experimental Study of the Antiactivation of Reduced \\ Pellets at Low Temperatures*
}

\section{By Yoshiaki IGUCHI I** $^{*}$ and Michio INOUYE**}

\section{Synopsis}

It is generally recognized that iron oxide pellets reduced by hydrogen at lower temperatures are easily reoxidized in an air atmosphere even at room temperature.

However, pellets slightly reoxidized by the inert gas of a lower oxygen potential showed inactive behaviours. Very thin oxide layer of the thickness less than $9 \AA$ was formed on the pore surface of the pellet during reoxidation with nitrogen-oxygen gas mixture of below $1 \%$ oxygen. When the oxygen content in the gas mixture was below $1 \%$, the degree of reoxidation was nearly $0.9 \%$ independent of the oxygen potential. But if the oxygen content exceeded $1 \%$, the degree of reoxidation increased rapidly with the oxygen content.

It was also shown that the rate of reoxidation was controlled by the diffusion of oxygen through the boundary and the reacted shell layers. Therefore, the exposure time necessary for antiactivation could be calculated and it was dependent on the oxygen potential.

Furthermore, when the resistance of the gas transport was negligibly small, the rate of reoxidation was represented by the logarithmic rate law.

\section{Introduction}

It is well known that the reduced pellets are easily reoxidized in the air at room temperature. Especially, the pellets reduced at lower temperatures ignite in the air.

Nevertheless, the reoxidation behaviours of reduced pellets have not yet been studied sufficiently. In order to prevent the ignition of sponge-iron powders reduced at lower temperatures, the treatment in an inert gas of extremely low oxygen potential is known ${ }^{1)}$ to be effective. But the necessary oxygen potential and the treating time for antiactivation have not been clarified yet. The authors investigated the reoxidation behaviours of reduced pellets in order to know the conditions necessary for the antiactivation and also to clarify the mechanism of such antiactivation.

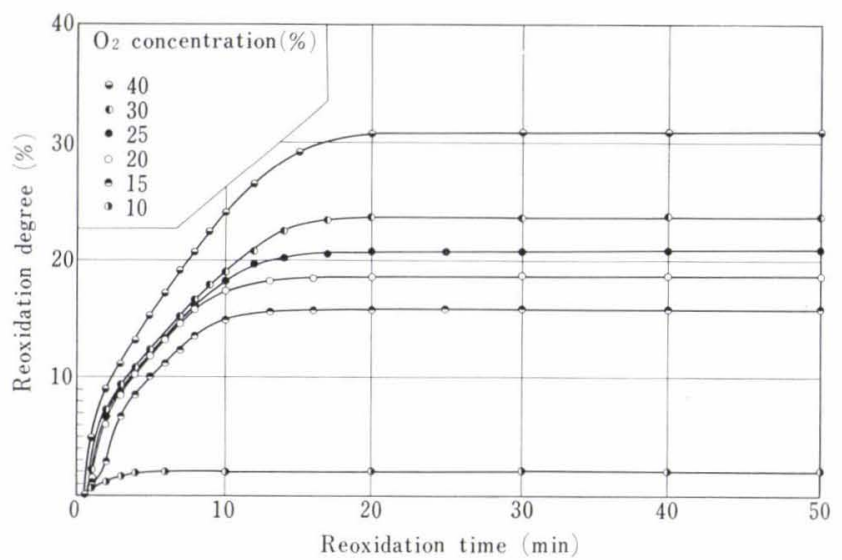

Fig. 1. Effect of the oxygen content on the reoxidation of reduced pellet (Oxygen content: over $10 \%$, temperature: $25^{\circ} \mathrm{C}$ )
From the view point of iron and steel industry, the pellets are generally reduced at higher temperatures, however, the pellets reduced at lower temperatures are particularly interested in the present study.

\section{Materials}

In the present work, all the experiments were carried out with hematite pellets prepared by balling of oxide particles and igniting at $1300^{\circ} \mathrm{C}$ for $6 \mathrm{hr}$ in the air. The porosity of the pellets was 18 to $28 \%$ and the pore surface areas were 11 to $13 \mathrm{sqm}$ per gram.

\section{Experimental Procedure}

All the rate measurements were made with the same balance system which had been used in the previous works. ${ }^{2)}$ The sensibility of the balance is $0.5 \mathrm{mg}$. The pellet was suspended from the balance in a reaction tube heated by an electric resistance furnace.

The reaction tube made of silica was $33 \mathrm{~mm}$ ID and $1000 \mathrm{~mm}$ long, and its lower part was packed with alumina particles to preheat the reaction gas. A $\mathrm{Pt}-\mathrm{Pt} \cdot 13 \% \mathrm{Rh}$ thermocouple covered with a $6 \mathrm{~mm}$ silica protection tube was inserted from the bottom of reaction tube to the position $5 \mathrm{~mm}$ below the sample.

The reduction and reoxidation of the pellets were carried out in series. The pellet was weighed, put in a basket made of platinum wire and suspended from the balance. While the pellet was heated to a desired reduction temperature, the atmosphere in the reaction tube was purged with nitrogen. Hydrogen was then introduced and the weight loss was recorded at adequate time intervals. After the pellet was completely reduced to sponge iron, it was cooled down to the reoxidation temperature in the same atmosphere and then the atmosphere in the reaction tube was

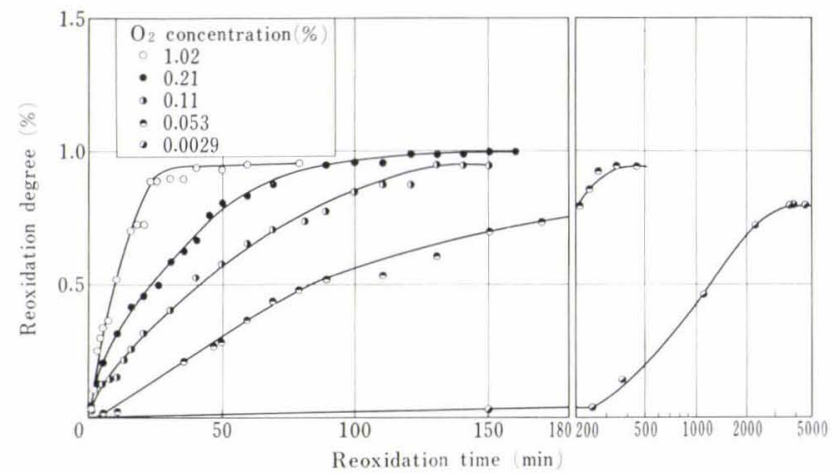

Fig. 2. Effect of the oxygen content on the reoxidation of reduced pellet (Oxygen content: below 1\%, temperature: $25^{\circ} \mathrm{C}$ )

* Originally published in Tetsu-to-Hagané, 58 (1972), 375, in Japanese. English version received December 12 , 1972.

** Department of Metallurgy, Faculty of Engineering, Nagoya University, Furo-cho, Chikusa-ku, Nagoya 464. 
purged with nitrogen. To remove oxygen contained in commercial grade nitrogen as an impurity, so as to prevent the slightest reoxidation, nitrogen from a cylinder was flowed through the packed bed of copper chips which was reduced frequently by introducing hydrogen. Accordingly, any oxygen could not be detected by gas chromatograph with high sensibility. Then, nitrogen containing some amount of oxygen was introduced and the weight increase was measured by the balance at adequate time intervals.

\section{Experimental Results}

1. Reoxidation of Reduced Pellets under Several Oxygen Potentials at Room Temperature

Pellets reduced by hydrogen at $520^{\circ} \mathrm{C}$ were reoxidized at room temperature with nitrogen containing various amounts of oxygen, $29 \mathrm{ppm}$ to $40 \%$. The reoxidation curves obtained are given in Figs. 1 and 2. As shown in these figures, if the reduced pellets are reoxidized with the gas of lower oxygen concentration, below $1 \%$ oxygen, the reoxidation does not proceed over $1.0 \%$ independent of the oxygen potential. On the other hand, in the case of oxygen potential higher than $5 \%$ oxygen, the reoxidation proceeds with increasing oxygen potential. It is also observed that the reoxidation proceeds up to $1.0 \%$ more rapidly with increasing oxygen potential. The detailed description on the relationship between the degree of reoxidation and the oxygen potential is referred to section $I V .5$. Here, the degree of reoxidation is defined as the percentage of oxidation up to ferric oxide.

\section{Heat Evolved by Reoxidation}

Since the oxidation of metallic iron is exothermic and the reduced pellet has a large reaction interface area, the temperature change during the reoxidation must be taken into account for the investigation of reoxidation behaviours.

When the raw pellet was prepared, the hot junction of $\mathrm{Pt}-\mathrm{Pt} \cdot 13 \% \mathrm{Rh}$ thermocouple was settled so as to be in the center of the pellet. Then the raw pellet was heated in the air at $1300^{\circ} \mathrm{C}$ for $6 \mathrm{hr}$.

The pellets prepared were reduced by hydrogen at $500^{\circ} \mathrm{C}$ and then reoxidized in various oxygen potentials at room temperature.

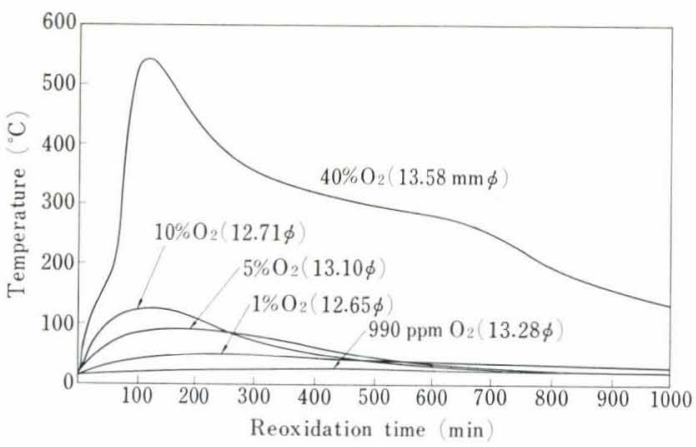

Fig. 3. Temperature changes of reduced pellets by reoxidation with nitrogen-oxygen gas mixture of various oxygen content. Figures in parentheses show diameters of pellets.
The temperature change was recorded automatically during reoxidation. The results are shown in Fig. 3 where the diameters of pellets are given by the number in parenthesis.

The highest peak of the temperature curve during the reoxidation became higher and sharper, and the time to reach the peak became shorter with increasing oxygen potential. Below $1 \%$ oxygen, the temperature rises were relatively small.

\section{Microscopic and X-ray Analysis of Reduced Pellets Reoxidized in Various Oxygen Potentials at Room Temperature}

In order to analyse the process of reoxidation, cross sections of the pellets reoxidized in various oxygen potentials were observed.

Macroscopic inspection of the fracture surface shows that an light gray inner core is surrounded by a dark gray outer shell of oxide layer, when the oxygen potential is higher than 5\%. A typical figure is shown in Photo. 1.

The thickness of the macroscopic oxide layer increases with increasing oxygen potential above 5\% oxygen. When the pellets were reoxidized in lower oxygen potentials such as below $1 \%$ oxygen, the cross section was observed to be apparently uniform like the core shown in the Photo. 1. The interface between the outer shell and the inner core seems to be parallel to the surface. This fact suggests that the rate of oxidation of metallic iron on the pore surface is rapid as compared with the diffusion rate of oxygen in the reoxidized shell layer. Therefore, the reoxidation of reduced pellets might proceed topochemically.

Furthermore, microscopic observation shows that the macroscopic oxide shell layer consists of the thin oxide film on the pore surface and the metallic iron inside of this film. In the inner core, however, any microscopic oxide layer could not be observed. The

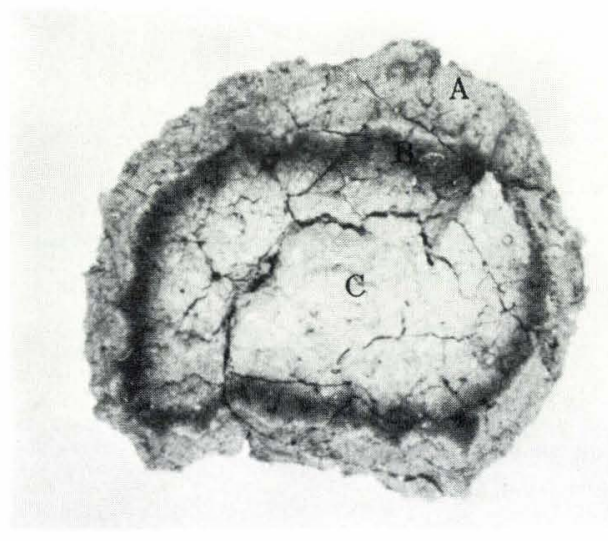

Photo, 1. Cross sectional view of a pellet reduced by hydrogen at $520^{\circ} \mathrm{C}$ and reoxidized by nitrogen-oxygen gas mixture at room temperature

A: Macroscopic oxide shell layer

B: Transitional shell layer

C: Inner core 
pellets reoxidized in the oxygen potential below $1 \%$ show no more microscopic oxide film. It was also clarified that the visual colour of the macroscopic oxide shell might vary with the thickness of microscopic oxide films.

The typical microphotograph of the macroscopic oxide shell is shown in Photo. 2.

The identification of metallic iron, wustite, magnetite and hematite in both parts was made by X-ray diffraction, using iron target, and the results obtained are listed in Table 1.

Hematite was detected only for the pellet reoxidized in such higher oxygen potential as $40 \%$ oxygen and magnetite was seen for any pellet reoxidized in the oxygen potential higher than $5 \%$. For the pellets reoxidized in the oxygen potential less than $1 \%$ and also the inner cores reoxidized in the oxygen potential higher than $5 \%$, nothing was observed except metallic iron.

\section{Reoxidation of Reduced Pellets at $100^{\circ}$ to $300^{\circ} \mathrm{C}$ under Lower Oxygen Potentials}

As mentioned in section $I V$. 1 , it is evident that if the reduced pellets are reoxidized to a definite degree under lower oxygen potentials, they are not further reoxidized.

It is generally recognized ${ }^{3)}$ that the rate of oxidation of metallic iron obeys the logarithmic rate law at lower temperatures and the parabolic rate law at higher temperatures. Concerning the transition temperature from the logarithmic to the parabolic rate law, a close agreement among earlier works was not necessarily obtained. However, it is obvious ${ }^{4)}$ that the

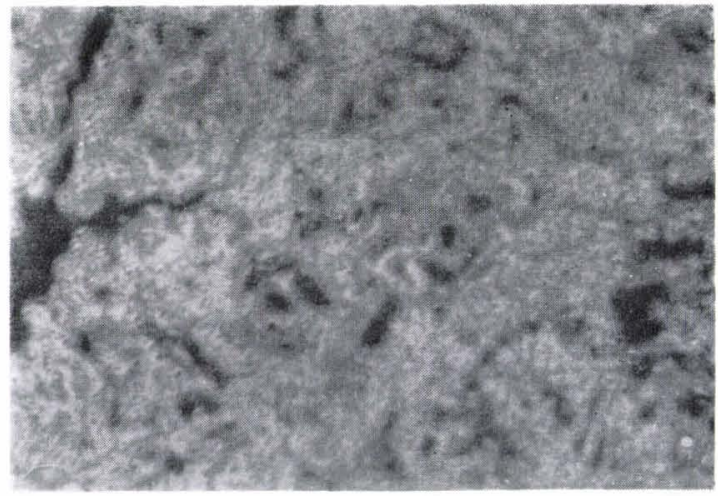

Photo. 2. Microphotograph of the oxide layer of a reoxidized (by $20 \%$ oxygen-nitrogen gas mixture) pellet which was reduced at $520^{\circ} \mathrm{C}$, showing microscopic oxide layer and metallic iron

Table 1. X-ray analysis of reduced pellets reoxidized by various oxygen concentration

\begin{tabular}{c|c|c}
$\begin{array}{c}\text { Partial pressure } \\
\text { of oxygen }(\mathrm{atm})\end{array}$ & Outside layer & Inner core \\
\hline 0.40 & $\mathrm{Fe}, \mathrm{Fe}_{3} \mathrm{O}_{4}, \mathrm{Fe}_{2} \mathrm{O}_{3}$ & $\mathrm{Fe}$ \\
0.30 & $\mathrm{Fe}, \mathrm{Fe}_{3} \mathrm{O}_{4}$ & $\mathrm{Fe}$ \\
0.25 & $\mathrm{Fe}, \mathrm{Fe}_{3} \mathrm{O}_{4}$ & - \\
0.20 & $\mathrm{Fe}, \mathrm{Fe}_{3} \mathrm{O}_{4}$ & $\mathrm{Fe}$ \\
0.10 & & $\mathrm{Fe}, \mathrm{Fe}_{3} \mathrm{O}_{4}$
\end{tabular}

logarithmic rate law is applicable to the initial stage of the oxidation of iron at the temperature in the neighbourhood of $\mathrm{A}_{1}$ transformation point of iron.

The authors ${ }^{5)}$ have already shown that the logarithmic rate law is valid to the reoxidation of iron powders reduced by hydrogen.

According to the logarithmic rate law, at room temperature the reoxidation proceeds very rapidly at first and its rate decreases after a definite period as if the further reoxidation was stopped. Therefore, the present results that the reoxidation of reduced pellets seems to stop at a definite degree of reoxidation may be resulted from the fact that the oxidation of metallic iron on the pore surface obeys the logarithmic rate law.

In order to ascertain whether or not the logarithmic rate law might be valid to the reoxidation of reduced pellets, the pellets reduced at $520^{\circ} \mathrm{C}$ were reoxidized

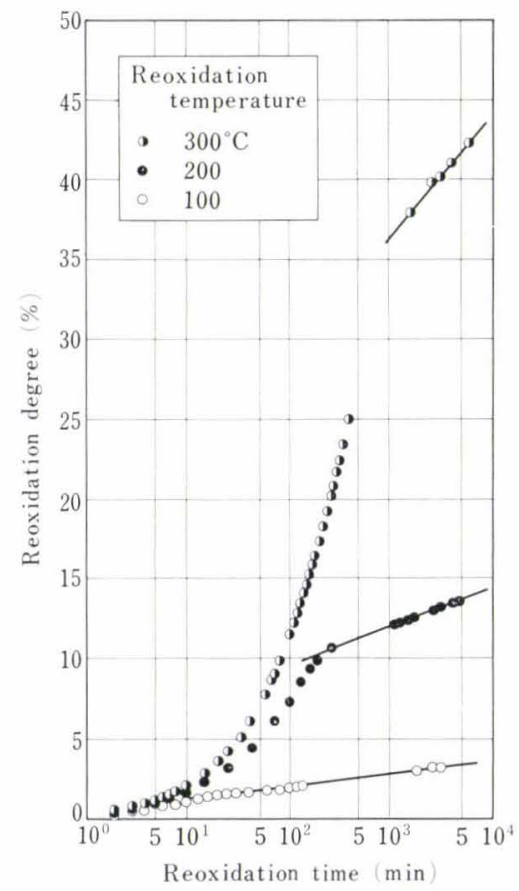

Fig. 4. Logarithmic plots of reoxidation curves of reduced pellets, reoxidized by nitrogen-oxygen gas mixture containing $1 \%$ oxygen at $100^{\circ}$ to $300^{\circ} \mathrm{C}$

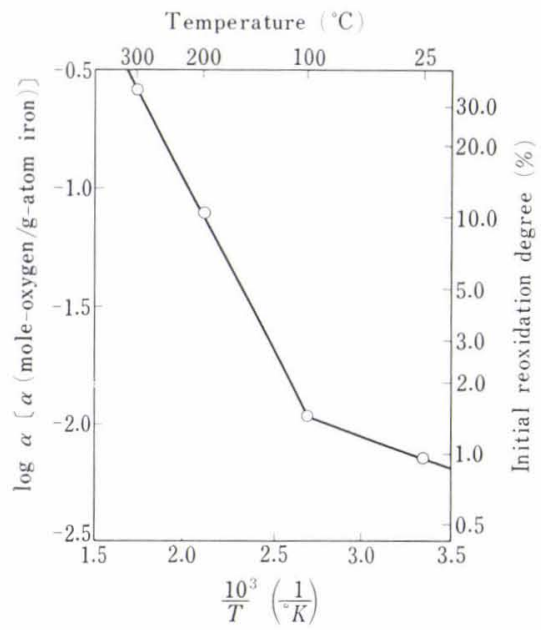

Fig. 5. Relations between the reoxidation temperature and the initial reoxidation degree of reduced pellet with gas containing $1 \%$ oxygen 
in the nitrogen containing $1 \%$ oxygen at $100^{\circ}, 200^{\circ}$, and $300^{\circ} \mathrm{C}$, and the results obtained are shown in Fig. 4 with a semi-log scale. Except the initial stage, it is clear that linear relationships may hold at each temperature. That is, the logarithmic rate law is applicable to the later stage. Now, it is defined that the degree of initial reoxidation is that at which the logarithmic rate law begins to hold. In Fig. 5, the degrees of initial reoxidation obtained from Fig. 4 are shown as Arrhenius-type plots. It shows that the degree of initial reoxidation increases with increasing reoxidation temperature, especially above $100^{\circ} \mathrm{C}$.

\section{Effect of the Oxygen Potential on the Degree of Reoxida- tion}

The relationship between the degree of initial reoxidation and the oxygen potential in the range of $29 \mathrm{ppm}$ to $40 \%$ oxygen is shown in Fig. 6. It is obvious that although the degree of initial reoxidation increases with oxygen concentration in the cases more than $5 \%$ oxygen, it becomes nearly constant at the oxygen concentration below $1 \%$ independent of the oxygen potential.

It is reasonable to consider that the degree of initial reoxidation increases with increasing oxygen potential, due to the temperature rise of the pellet by the heat of oxidation which accelerates reoxidation. If the oxygen potential is lower than $1 \%$, the temperature rise is negligibly small and its effect on the reoxidation becomes also negligible. Therefore, it seems that the degree of initial reoxidation is nearly constant below $1 \%$ oxygen at room temperature. Even in the oxygen potential above $5 \%$, if the temperature rise could be prevented, the degree of reoxidation would not increase and show the similar values as in the case of lower oxygen potential below $1 \%$.

Considering that the inner structures of the reoxidized pellets in the oxygen potential below $1 \%$ are observed to be uniform microscopically, it seems that any part of the pellet should absorb a definite content of oxygen and show no more increase of reoxidation.

Since the degree of reoxidation depends on the temperature rise during the reoxidation, the range of the oxygen potential where the degree of reoxidation is constant may also depend on the characteristics of heat conduction surrounding the sample such as the heat conductivity of gas.

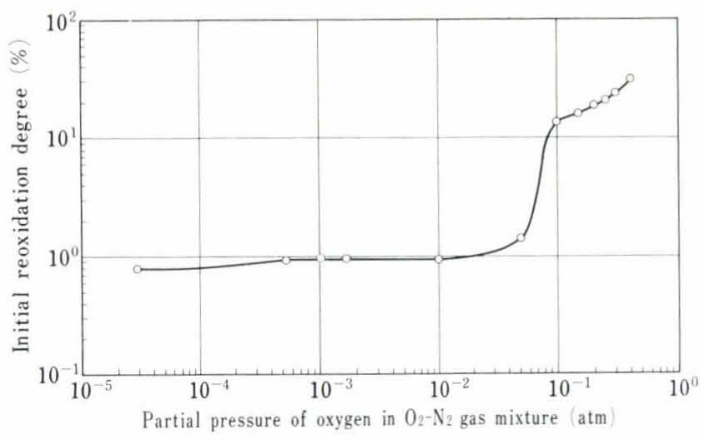

Fig. 6. Relation between the reoxidation degree of reduced pellet (reduced at $520^{\circ} \mathrm{C}$ ) and the oxygen content at room temperature
It was also recognized that the pellet reoxidized in a lower oxygen potential was not further reoxidized even in a higher oxygen potential. Consequently, the pellets slightly reoxidized in the oxygen potential below $1 \%$ are regarded as to be antiactivated.

\section{Antiactivation of the Pellets Reduced at Various Tem- peratures}

It is quite clear that the pellets reduced at $520^{\circ} \mathrm{C}$. are antiactivated by reoxidizing in the low oxygen potential below $1 \%$ oxygen. Now, in order to ascertain the effects of reducing temperature on the degree of initial reoxidation, the pellets reduced at a temperature in the range of $400^{\circ}$ to $690^{\circ} \mathrm{C}$ were reoxidized in the oxygen potential of $0.1 \%$ oxygen. The results are shown in Fig. 7 in which the degree of reoxidation decreases rapidly with increasing reduction temperature below $600^{\circ} \mathrm{C}$ but gradually decreases above $600^{\circ} \mathrm{C}$.

7. Amount of Oxygen Absorbed per Unit Surface Area of the Pellets Reduced at Various Temperatures

In order to obtain the amount of oxygen absorbed

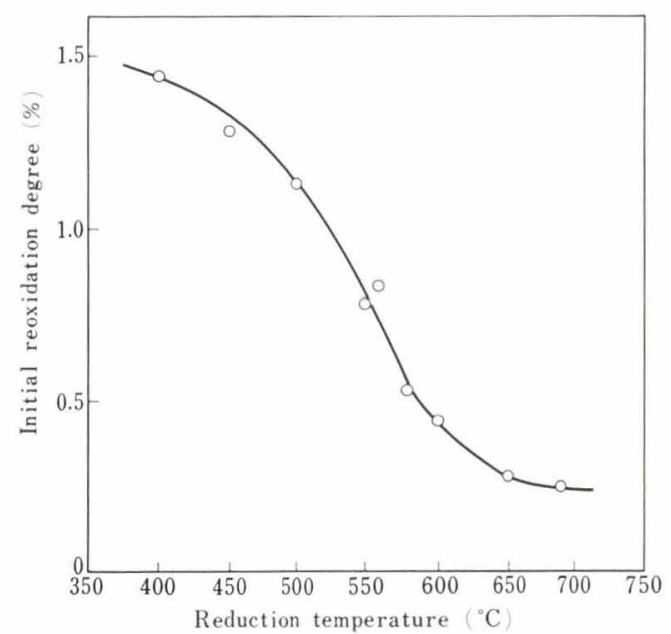

Fig. 7. Relation between the reoxidation degree of reduced pellet and the reduction temperature

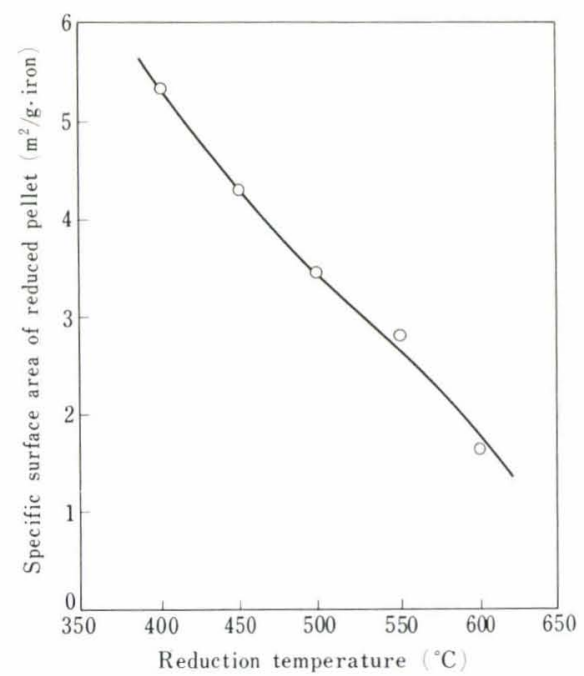

Fig. 8. Specific surface areas of pellets reduced at various temperatures 
per unit surface area of reduced pellets, specific surface areas of the pellets reduced at various temperatures were measured by applying the BET equation for adsorption isotherms of nitrogen at $-195^{\circ} \mathrm{C}$. The relationship between the specific surface area and reduction temperature is shown in Fig. 8 in which the area decreases linearly with increasing reduction temperature. This is in good agreement with the previous work. ${ }^{5)}$

Now, if the composition of oxide layers could be analysed, the thickness of such layers would be calculated from the amount of oxygen absorbed per unit area. However, this composition can not be determined by $\mathrm{X}$-ray analysis as described in section $I V$. 3 , and the thickness of oxide layer was therefore calculated by Eq. (1), assuming the oxide layer to be a simple iron oxide, that is, wustite, magnetite, or hematite where their densities are $5.261 \mathrm{~g} / \mathrm{cm}^{3}, 5.136$ $\mathrm{g} / \mathrm{cm}^{3}$ and $5.73 \mathrm{~g} / \mathrm{cm}^{3},{ }^{3)}$ respectively.

$$
x \cdot 10^{-8} \cdot d_{\text {oxide }} \cdot \frac{y M_{\mathrm{O}}}{M_{\mathrm{Fe}}+y M_{\mathrm{O}}}=\frac{100 \cdot \frac{F M_{\mathrm{O}}}{2 M_{\mathrm{Fe}}}}{S_{w}}
$$

The results are shown in Fig. 9. The maximum thickness is obtained when the pellets are reduced at nearly $520^{\circ} \mathrm{C}$ independent of the kind of iron oxide, and the value obtained is approximately $9 \AA$.

\section{Discussions}

As described in section $I V$. 4, the logarithmic rate law is valid for the later stage of the reoxidation of reduced pellets as the oxidation of metallic iron. Accordingly, if the oxygen supply is enough to the reaction interface, the reoxidation rate of the reduced pellet would also obey the same logarithmic rate law. Since the reduced pellet, however, is porous and has a large specific surface area, oxygen molecule must diffuse inside the pores from the gas bulk to reach the reaction interface. Furthermore, the reduced pellets are more strongly affected by the exothermic reaction of

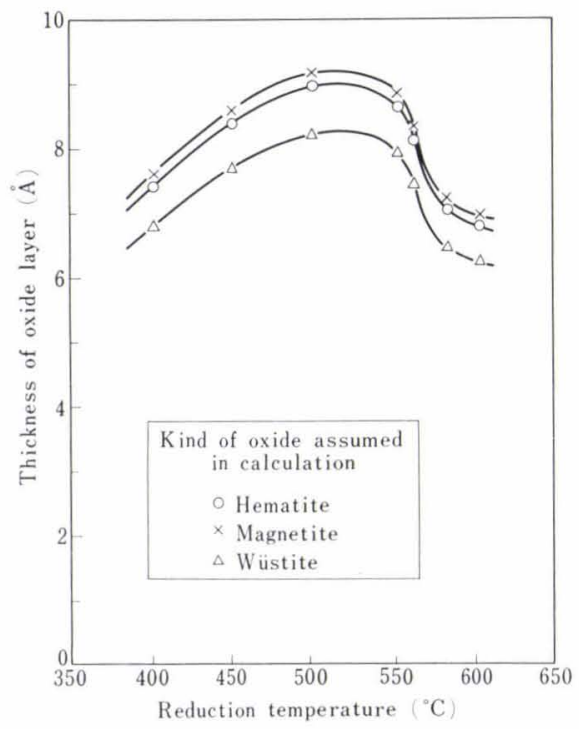

Fig. 9. Relation between the reduction temperature of pellet and the calculated thickness of oxide layer of reduced pellets, reoxidized by gas containing $0.1 \%$ oxygen at room temperature oxidation due to the large reaction interface. Those factors are different from the oxidation behaviour of metallic iron. In the present work, the analyses of the reoxidation process were carried out when a remarkable temperature increase of the pellet was not observed by reoxidation in a low oxygen potential.

\section{Reoxidation Rate of Metallic Iron on the Pore Surface of Reduced Pellets}

It is reasonable to consider that the oxidation rate law of metallic iron should also be applied to the reoxidation of metallic iron on the pore surface of a reduced pellet. In our previous work, ${ }^{5)}$ it was shown that the logarithmic rate law was valid to the later stage of the reoxidation of reduced iron-powders, and it was also valid for the reduced pellets, as described in section $I V$. 4. It is generally accepted that the logarithmic rate law is valid to the oxidation of metallic iron at lower temperatures and also to the initial period of the oxidation at higher temperatures.

According to the Mott's theory, ${ }^{6)}$ the logarithmic rate law can be applied to the oxidation of metals whose oxides are ion conductors and also electron insulators. Namely, the rate of oxidation is limited by the rate of electron transfer. Electrons can penetrate through the oxide layer by tunnelling effect of quantum mechanics without going over the energy barrier. The probability of passing through is represented by $\exp \left(-x / x_{0}\right)$. The rate of growth of oxide layer is proportional to this probability and is represented by Eq. (2).

$$
d x / d t \propto \exp \left(-x / x_{0}\right)
$$

Integrating Eq. (2), Eq. (3) is obtained

$$
x=x_{0} \log \left(t+t_{0}\right)-x_{0} \log t_{0}
$$

When $t \gg t_{0}$, Eq. (3) becomes

$$
x=x_{0} \log t-x_{0} \log t_{0}
$$

Substituting Eq. (1) into Eq. (4), it becomes

$$
\begin{array}{r}
F=k \log t-k \log t_{0} \ldots \ldots \ldots \ldots \\
k=S_{w} \cdot d_{\text {oxide }} \cdot \frac{y M_{\mathrm{Fe}}}{M_{\mathrm{Fe}}+y M_{\mathrm{O}}} \cdot \frac{2}{3} \cdot 10^{-6} \cdot x_{0}
\end{array}
$$

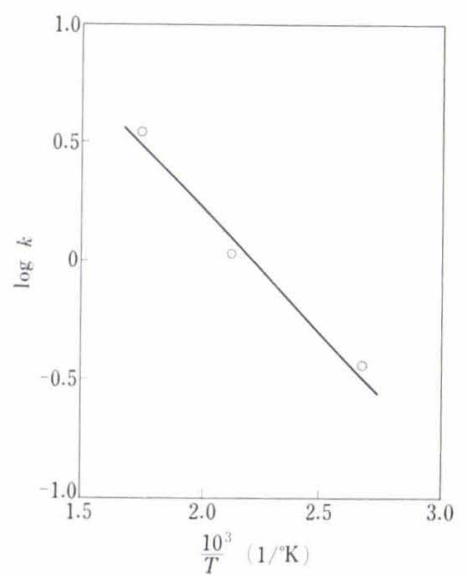

Fig. 10. Temperature dependence of the rate constant for reoxidation of reduced pellet as shown by the logarithmic law 
Since the increase of the weight is approximately proportional to the thickness of the oxide layer, it is linearly proportional to the logarithmic value of reoxidation time. The results in the present work satisfy the logarithmic rate law, as mentioned in section $I V$. 4.

It is suggested from the above theory that $x_{0}$ depends on the temperature, but not on the oxygen potential. Applying the results shown in Fig. 4, the values of $k$ were estimated as $0.363,1.08$, and 3.50 (-), respectively. They satisfied approximately the Arrhenius-type relation, as shown in Fig. 10. The apparent activation energy was estimated to be $4.8 \mathrm{kcal} /$ mol which was slightly larger than the value obtained for the reoxidation of reduced iron-powders which was $3 \mathrm{kcal} / \mathrm{mol}$, but remarkably less than that for the oxidation of iron plate, $21.7 \mathrm{kcal} / \mathrm{mol}$, reported by Measor et al. ${ }^{4)}$

\section{Reoxidation Rate of Reduced Pellets}

As described in section $I V$. 4, the reoxidation process of the reduced pellets is analysed by separating into the initial stage and the later one. Here, the present results show that the logarithmic rate law can be applied to the later stage. It is generally recognized that this law is applicable to the oxidation of metallic iron at lower temperatures and to the initial period of the oxidation of metallic iron at higher temperatures.

According to the logarithmic rate law, a metallic iron is rapidly oxidized to a definite degree of reoxidation. In this case, the rate of reoxidation reaction of metallic iron on the pore surface may be very rapid.$^{6}$

Therefore, it is concluded that the rate limiting step in the initial stage of the reoxidation of reduced pellets should be the gas diffusion process both in the boundary layers and in the reoxidized shell layers of reduced pellets, or the both processes of the gas diffusion and the reoxidation of the pore surface, but not the oxidation process on the pore surface.

Furthermore, as described in section $I V .1$, the rate of initial reoxidation of reduced pellets increases with increasing oxygen potential. This fact also suggests that the initial stage of the reoxidation of reduced pellets is mainly controlled by the gas diffusion, and that the rate constant, $k$, is independent of oxygen potential.

It is well known ${ }^{7)}$ that the gas-solid reaction proceeds topochemically when the rate of chemical reaction at the interface is remarkably rapid and the gas diffusion is rate contolling. This is comfirmed by the macroscopic observation of the cross sections of reduced pellets reoxidized in the oxygen potential above $5 \%$.

If it is assumed that the reoxidation rate of the reduced pellet should be controlled by the gas diffusion in the gas boundary and the reoxidized shell layers, the rate equation can be given by Eq. (7), where the reoxidation model is shown in Fig. 11.

$$
\begin{aligned}
\frac{1}{D_{e}} & {\left[\frac{1}{2}\left\{1-(1-R)^{2 / 3}\right\}-\frac{1}{3} R\right]+\frac{R}{3 k_{f} r_{0}} } \\
& =\frac{C_{\mathrm{O}_{2}}^{b}-C_{\mathrm{O}_{2}}^{r}}{d_{\mathrm{iron}} \cdot \alpha \cdot r_{0}^{2}} \cdot t \ldots \ldots \ldots \ldots \ldots \ldots \ldots \ldots \ldots \ldots \ldots \ldots \ldots
\end{aligned}
$$

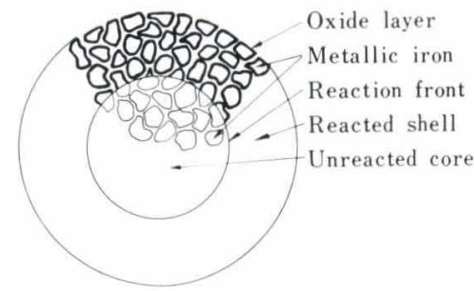

Fig. 11. Reoxidation model

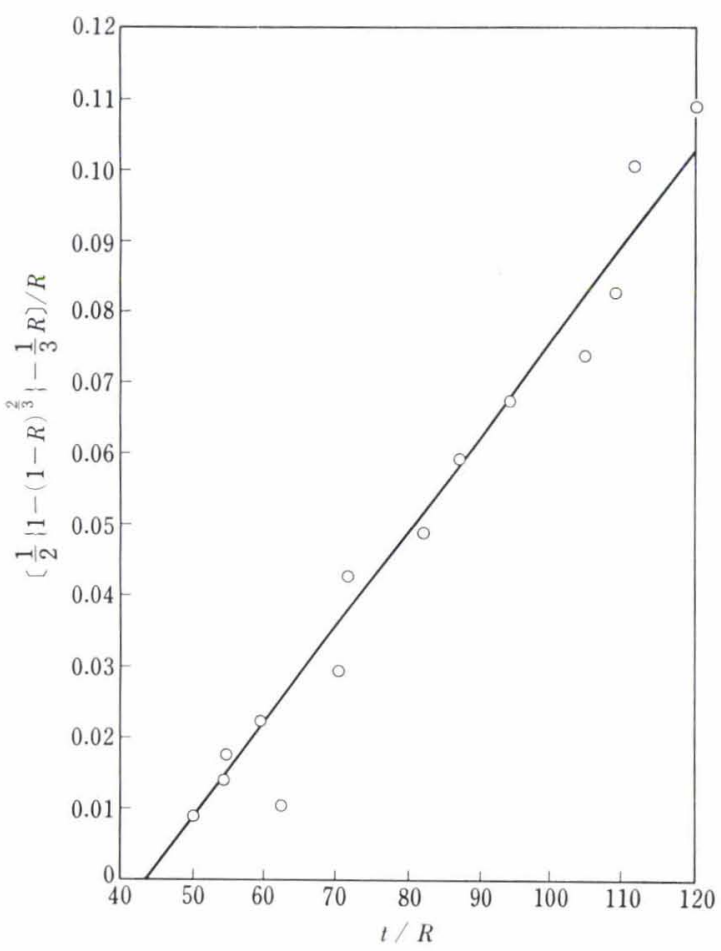

Fig. 12. Relation between $\left[\frac{1}{2}\left\{1-(1-R)^{2 / 3}\right\}-\frac{1}{3} R\right] / R$ and $t / R$ shown in Eq. (11) for No. 3

Since oxygen molecules react with iron atoms and disappear at the reaction interface, the number of molecules in the gas phase decreases at the reaction interface. Hence, bulk flow occurs from the bulk gas phase toward the reaction interface.

The rate of oxygen transfer by bulk flow and diffusion can be calculated from the following equation, assuming quasi-stationary state.

$$
J_{\mathrm{O}_{2}}^{d}+J_{\mathrm{O}_{2}}^{c}=C_{0} \cdot \frac{D_{e}}{r_{0}} \cdot \frac{r}{r-r_{0}} \cdot \ln \frac{C_{0}}{C_{\mathrm{N}_{2}}^{r_{0}}}
$$

On the other hand, the rate of diffusion through shell layer is given by Eq. (9), neglecting bulk flow.

$$
J_{\mathrm{O}_{2}}^{d}=\frac{D_{e}}{r_{0}} \cdot \frac{r}{r-r_{0}} \cdot C_{0_{2}}^{r_{0}} \cdot \cdots
$$

The ratio of the diffusion flux to the total transfer flux is expressed by Eq. (10).

$$
J_{\mathrm{O}_{2}}^{d} /\left(J_{\mathrm{O}_{2}}^{d}+J_{\mathrm{O}_{2}}^{c}\right)=\frac{C_{\mathrm{O}_{2}}^{r_{0}}-C_{\mathrm{O}_{2}}^{r}}{C_{0} \ln \left(C_{0} / C_{\mathrm{N}_{2}}^{r_{0}}\right)}
$$

The relation given in Eq. (10) shows that $J_{\mathrm{O}_{2}}^{d} /$ $\left(J_{\mathrm{O}_{2}}^{d}+J_{\mathrm{O}_{2}}^{c}\right)$ may gradually approach unity, namely $J_{\mathrm{O}_{2}}^{c}$ may approach to zero, when the concentration of oxygen $C_{O_{2}}^{r_{0}}$ approaches to zero. The transfer of oxy- 
Table 2. Effective diffusion coefficient $D_{e}$ and mass transfer coefficient $k_{f}$ in the reoxidation of pellets

\begin{tabular}{|c|c|c|c|c|c|c|c|c|c|}
\hline No. & $C_{0_{\mathrm{g}}}^{b}\left(\mathrm{~mol} / \mathrm{cm}^{3}\right)$ & $\begin{array}{c}\alpha \text { (mol-oxygen/ } \\
\text { g-atom-iron) }\end{array}$ & $r_{0}(\mathrm{~cm})$ & $\begin{array}{c}d_{\text {iron }} \\
\left(\text { g-atom } / \mathrm{cm}^{3}\right)\end{array}$ & $\begin{array}{c}D_{e} \\
\left(\mathrm{~cm}^{2} / \mathrm{sec}\right)\end{array}$ & $D_{e} / D_{\mathrm{N}_{2}-\mathrm{O}_{2}}$ & $\begin{array}{c}k_{f}^{\operatorname{expt}} \\
(\mathrm{cm} / \mathrm{sec})\end{array}$ & $\begin{array}{l}k_{f}^{\mathrm{calc}}(\mathrm{R} . \mathrm{M} .) \\
(\mathrm{cm} / \mathrm{sec})\end{array}$ & $\begin{array}{c}k_{f}^{\mathrm{calc}}(\mathrm{S} . \mathrm{T} .) \\
(\mathrm{cm} / \mathrm{sec})\end{array}$ \\
\hline 1 & $4.18 \times 10^{-7}$ & $7.09 \times 10^{-3}$ & 0.662 & $5.16 \times 10^{-2}$ & 0.054 & 0.25 & 0.26 & 0.809 & 0.728 \\
\hline 2 & $8.61 \times 10^{-8}$ & $7.15 \times 10^{-3}$ & 0.620 & $5.05 \times 10^{-2}$ & 0.079 & 0.37 & 0.81 & 0.847 & 0.760 \\
\hline 3 & $4.51 \times 10^{-8}$ & $8.48 \times 10^{-3}$ & 0.633 & $4.74 \times 10^{-2}$ & 0.079 & 0.37 & 0.72 & 0.835 & 0.749 \\
\hline 4 & $2.17 \times 10^{-8}$ & $6.93 \times 10^{-3}$ & 0.608 & $5.04 \times 10^{-2}$ & 0.065 & 0.31 & 0.51 & 0.843 & 0.756 \\
\hline 5 & $1.19 \times 10^{-9}$ & $5.92 \times 10^{-3}$ & 0.576 & $5.08 \times 10^{-2}$ & 0.067 & 0.32 & 1.10 & 0.892 & 0.803 \\
\hline
\end{tabular}

gen by bulk flow may be practically negligible at the oxygen potential less than $1 \%$.

As mentioned above, it is observed that any part of the reduced pellet is reoxidized to a definite degree of reoxidation.

Oxygen potential in equilibrium with iron and magnetite, $1.63 \times 10^{-92} \mathrm{~mol} / \mathrm{cm}^{3}$, was substituted into $C_{\mathrm{O}_{2}}^{r}$ which was negligibly small as compared with $C_{\mathrm{O}_{2}}^{b}$. Then, the rate equation becomes as follows.

$$
\begin{aligned}
D_{e} & {\left[\frac{1}{2}\left\{1-(1-R)^{23}\right\}-\frac{1}{3} R\right]+\begin{array}{c}
R \\
3 k_{f} r_{0}
\end{array} } \\
= & \frac{C_{\mathrm{O}_{2}}^{d}}{d_{\mathrm{iron}} \cdot \alpha \cdot r_{0}^{2}} \cdot t \ldots \ldots \ldots \ldots \ldots \ldots \ldots \ldots \ldots \ldots \ldots \ldots
\end{aligned}
$$

Dividing Eq. (11) with $R$, Eq. (12) is obtained.

$$
\frac{\frac{1}{2}\left\{1-(1-R)^{2 / 3}\right\}-\frac{1}{3} R}{R}=\frac{D_{e} \cdot C_{\mathrm{O}_{2}}^{b}}{d_{\mathrm{iron}} \cdot \alpha \cdot r_{0}^{2}} \cdot \frac{t}{R}-\frac{D_{e}}{3 k_{f} r_{0}}
$$

When the gas diffusion through either the reoxidized shell or the gas boundary layer, or through both of them may control the rate of reoxidation, there is a linear relation between $\left[1 / 2\left\{1-(1-R)^{2 / 3}-1 / 3 R\right] / R\right.$ and $t / R$. Effective diffusivity and mass transfer coefficient can be calculated from the slope and the intersection of the straight line obtained by Eq. (12). The reoxidation curves in the present work satisfied the relation of Eq. (12) and shows a linear relationship, as shown in Fig. 12 .

The calculated values of $D_{e}$ and $k_{f}$ are listed in Table 2. It is obvious from this table that $D_{e}$ and $k_{f}$ are approximately constant independent of the oxygen potential.

Applying the value of the gas diffusivity in the system $\mathrm{N}_{2}-\mathrm{O}_{2}, \quad D_{\mathrm{N}_{2}-\mathrm{O}_{2}}=0.212 \mathrm{~cm}^{2} / \mathrm{sec}^{8)}$ at $25^{\circ} \mathrm{C}$, the values of $D_{e} / D_{\mathrm{N}_{2}-\mathrm{O}_{2}}$ are also calculated and are shown in Table 2.

Concerning the value of $k_{f}$, it can also be calculated from the correlations of dimensionless numbers, that is, Ranz-Marshall relation shown in Eq. (13) and Steinberger-Treyball relation given by Eq. (14).

$$
\begin{gathered}
S h=2.0+0.6 \operatorname{Re}^{1 / 2} \cdot S c^{1 / 3} \ldots \ldots \\
S h=2.0+0.343 \operatorname{Re}^{0.62} \cdot S c^{0.41}
\end{gathered}
$$

The results are listed in Table 2 together with the above values.

\section{Conclusions}

The pellets reduced at lower temperatures ignite if they are exposed to an air atmosphere, as the sponge iron powders reduced at the similar condition. In order to reveal the reoxidation phenomena and to prevent the ignition, the reoxidation of reduced pellets was investigated at room temperature over the range of oxygen potential, $29 \mathrm{ppm}$ to $40 \%$.

The reoxidation behaviours of the reduced pellets are different from the oxidation of metallic iron. Namely, the oxygen molecules must diffuse through the pore to reach the pore surface where the reaction occurs and also the reoxidation is strongly influenced by the temperature increase due to the heat of oxidation itself, as the specific reaction interface is larger than the metallic iron plate. In the present work, the following conclusions were obtained from the analysis of the rate of reoxidation for the former case.

(1) If the reduced pellets are reoxidized to a definite degree of reoxidation further reoxidation does not proceed even in the higher oxygen potentials.

(2) While the degree of final reoxidation increases with increasing oxygen potential when the potential is higher than $5 \%$ oxygen, it shows, however, constant values independent of the oxygen potential in the case lower than $1 \%$. The degree of reoxidation for a lower oxygen potential may be determined by the characteristics of the reduced pellet.

(3) The results that the degree of reoxidation increases with increasing oxygen potential above 5\%, could be interpreted by the temperature rise due to the heat of reaction which promotes the reoxidation, especially above $100^{\circ} \mathrm{C}$. The degree of reoxidation would show the definite value if the temperature rise could be prevented by the sufficiently rapid cooling.

(4) The rate of reoxidation is controlled by the gas diffusion through the gas boundary and the reoxidized shell layers. Therefore, the time required for the antiactivation of reduced pellets can be calculated from the model for gas diffusion controlling.

(5) The results that the reoxidation seems to stop after a slight reoxidation up to a definite degree in the lower oxygen potential such as below $1 \%$ would be interpreted by the logarithmic rate law which is valid for the rate of reoxidation.

(6) The macroscopic oxide shell layer is formed when the pellets are reoxidized in the oxygen potential above $5 \%$. This oxide shell layer is composed of the microscopic oxide film and the metallic iron remained beneath the film. The macroscopic inner cores are apparently composed of the metallic iron alone, so far as by X-ray and microscopic examinations, as observed on the pellets reoxidized below $1 \%$ oxygen. 


\section{Appendix}

Jost $^{9)}$ has derived the rate equation of bulk flow and diffusion for the gas-solid reaction.

The rate equations for bulk flow and diffusion in the reaction (1) have been derived under the boundary conditions of reoxidation.

$$
S_{1}+G_{1} \longrightarrow S_{2}
$$

Now, consider the system in which gas molecules reach the reaction interface and react with solid reactant as described by the reaction (1).

Here, $r$ is defined as the radius of unreacted core of a pellet which has the radius $r_{0}$ and $\rho$ is also defined as the radial variable. Since gas molecules consumes at the reaction interface, gaseous reactant is transported to the interface by both diffusion and bulk flow. The flow rates are derived by assuming the quasi-stationary state.

The convection of gas flow in the direction of $+\rho$ is given by Eq. (2)

$$
v \rho^{2}=v_{0} r_{0}^{2}, \quad v=v_{0} \cdot \frac{r_{0}^{2}}{\rho^{2}}
$$

The diffusion rate is represented by Eq. (3),

$$
J_{1}^{d}=-D_{e} \cdot \frac{\partial C_{1}}{\partial \rho}, \quad J_{2}^{d}=-D_{e} \cdot \frac{\partial C_{2}}{\partial \rho}=D_{e} \cdot \frac{\partial C_{1}}{\partial \rho}
$$

and the flow rate due to the bulk flow is also shown by Eq. (4).

$$
J_{1}^{c}=C_{1} v=C_{1} v_{0} \cdot \frac{r_{0}^{2}}{\rho^{2}}, \quad J_{2}^{c}=C_{2} v=C_{2} v_{0} \cdot \frac{r_{0}^{2}}{\rho^{2}}
$$

Therefore, the total flow is expressed by Eq. (5)

$$
\begin{array}{r}
J_{1}=-D_{e} \cdot \frac{\partial C_{1}}{\partial \rho}+C_{1} v_{0} \cdot \frac{r_{0}^{2}}{\rho^{2}} \ldots \ldots \\
J_{2}=D_{e} \cdot \frac{\partial C_{1}}{\partial \rho}+C_{2} v_{0} \cdot \frac{r_{0}^{2}}{\rho^{2}} \ldots \ldots \ldots \\
J_{1}+J_{2}=\left(C_{1}+C_{2}\right) \cdot v_{0} \cdot \frac{r_{0}^{2}}{\rho^{2}}=C_{0} v_{0} \cdot \frac{r_{0}^{2}}{\rho^{2}}
\end{array}
$$

Taking the mass balance within the shell layer of radius $\rho$ and thickness $d \rho$, the time-dependent diffusion equation for component 1 is

$$
\begin{aligned}
\frac{\partial C_{1}}{\partial t} & =-\operatorname{div}\left(J_{1}\right) \\
& =D_{e}\left[\frac{\partial^{2} C_{1}}{\partial \rho^{2}}+\frac{2}{\rho} \cdot \frac{\partial C_{1}}{\partial \rho}\right]-\frac{\nu_{0} r_{0}^{2}}{\rho^{2}} \cdot \frac{\partial C_{1}}{\partial \rho}=0 \ldots
\end{aligned}
$$

Here, $\partial C_{1} / \partial t$ is equal to zero for a quasi-stationary state.

In the case $C_{1}$ depends on $\rho$ alone and $\mathrm{Eq}$. (7) is obtained instead of Eq. (6)

$$
\rho^{2} C_{1}^{\prime \prime}+C_{1}^{\prime}(2 \rho-\beta)=0
$$

where dashes denote differentiation with respect to $\rho$ and the length $\beta$ is given by Eq. (8).

$$
\beta=v_{0} r_{0}^{2} / D_{e}
$$

Eq. (7) can be solved under the following boundary conditions:

$$
\begin{gathered}
\left(J_{2}\right)_{0}=0, \quad \rho=r \\
C_{1}=0, \quad \rho=r \ldots \\
\left(J_{1}\right)_{r_{0}}=\left(J_{1}\right)_{r} \cdot \begin{array}{l}
r^{2} \\
r_{0}^{2}
\end{array}
\end{gathered}
$$

Substituting Eq. (5b) into Eq. (9a), then

$$
D_{e}\left(\begin{array}{c}
\partial C_{1} \\
\partial \rho
\end{array}\right)_{r}=-C_{2}^{r} \cdot v_{0} \cdot \begin{gathered}
r_{0}^{2} \\
r^{2}
\end{gathered}
$$

and substituting Eq. (5a) into Eq. (9c) and using Eq. (9b), Eq. (11) is obtained.

$$
-D_{e}\left(\begin{array}{c}
\partial C_{1} \\
\partial \rho
\end{array}\right)_{r_{0}}+C_{1}^{r_{0}} \cdot v_{0}=-D_{e}\left(\begin{array}{c}
\partial C_{1} \\
\partial \rho
\end{array}\right) \cdot r \begin{array}{r}
r^{2} \\
r_{0}^{2}
\end{array}
$$

Putting $C_{1}^{\prime}$ in Eq. (7) equal to $\xi$, then

$$
d \ln \xi=\beta-2 \rho
$$

and integrating between $r_{0}$ and $\rho$, Eq. (13) is obtained.

$$
\begin{gathered}
\ln \frac{\xi}{\xi_{0}}=\frac{\beta}{r_{0}}-\frac{\beta}{\rho}-2 \ln \frac{\rho}{r_{0}} \ldots \\
\xi \\
\xi_{0}=\left(\frac{r_{0}}{\rho}\right)^{2} \exp \left(\begin{array}{c}
\beta \\
r_{0}
\end{array}\right) \exp \left(-\frac{\beta}{\rho}\right)
\end{gathered}
$$

Substituting Eq. (8) into Eq. (13a), then

$$
\left(\begin{array}{c}
\partial C_{1} \\
\partial \rho
\end{array}\right)_{r}=\left(\begin{array}{c}
\partial C_{1} \\
\partial \rho
\end{array}\right)_{r_{0}} \cdot \begin{aligned}
& r_{0}^{2} \\
& r^{2}
\end{aligned} \exp \left(\begin{array}{c}
v_{0} r_{0} \\
D_{e}
\end{array}\right) \exp \left(-\begin{array}{c}
v_{0} r_{0}^{2} \\
r D_{e}
\end{array}\right) \ldots
$$

From Eqs. (10) and (11), the following equation is obtained.

$$
D_{e}\left(\begin{array}{c}
\partial C_{1} \\
\partial \rho
\end{array}\right)_{r_{0}}=C_{1}^{r_{0} v_{0}--} C_{2}^{r} \cdot v_{0}=-C_{2}^{r_{0}} \cdot v_{0}
$$

From Eqs. (11) and (14), Eq. (16) is obtained.

$$
C_{1}^{r_{0} \cdot v_{0}}=D_{e}\left(\frac{\partial C_{1}}{\partial \rho}\right)_{r_{0}}\left[1-\exp \left(\begin{array}{c}
v_{0} r_{0} \\
D_{e}
\end{array}\right) \exp \left(-\frac{v_{0} r_{0}^{2}}{r D_{e}}\right)\right]
$$

Substituting Eq. (16) into Eq. (15), it becomes

$$
C_{1}^{r_{0}} \cdot v_{0}=-C_{2}^{r_{0}} \cdot v_{0}\left[1-\exp \left(\begin{array}{c}
v_{0} r_{0} \\
D_{e}
\end{array}\right) \exp \left(-\begin{array}{c}
v_{0} r_{0}^{2} \\
r D_{e}
\end{array}\right)\right]
$$

Solving Eq. (17) for the exponentials and taking logarithms, Eqs. (18) and (19) are finally obtained.

$$
\begin{gathered}
v_{0} r_{0}-\frac{v_{0} r_{0}^{2}}{D_{e}}=\ln \frac{C_{1}^{r_{0}}+C_{2}^{r^{0}}}{C_{e}} \\
C_{2}^{r^{0}} \\
v_{0}=\frac{D_{e}}{r_{0}} \cdot \frac{r}{r-r_{0}} \cdot \ln \frac{C_{1}^{r^{0}}+C_{2}^{r^{0}}}{C_{2}^{r^{0}}}
\end{gathered}
$$

Changing $\rho$ into $r_{0}$ and substituting Eq. (15) into Eq. (19), Eq. (20) is obtained.

$$
J_{1}=-D_{e}\left(\frac{\partial C_{1}}{\partial \rho}\right)_{r_{0}}+C_{1}^{r_{0}} \cdot v_{0}=\left(C_{1}^{r_{0}}+C_{2}^{r_{0}}\right) \cdot v_{0} \quad \cdots
$$


and substituting Eq. (19) into Eq. (20), one obtaines

$$
J_{1}=C_{0} \cdot \frac{D_{e}}{r_{0}} \cdot \frac{r}{r-r_{0}} \ln \frac{C_{0}}{C_{2}^{r^{0}}}
$$

Next, the flow rate can be calculated by the diffusion alone, neglecting bulk flow. Eq. (22) is obtained from Eq. (3).

$$
J_{1}^{d}={ }_{r_{0}}^{D_{e}} \cdot \frac{r}{r-r_{0}} \cdot\left(C_{1}^{r}-C_{1}^{r_{0}}\right)
$$

Substituting Eq. (9b) into Eq. (22), Eq. (23) is obtained.

$$
J_{1}^{d}=\frac{D_{e}}{r_{0}} \cdot \frac{r}{r-r_{0}} \cdot C_{1}^{r_{0}}
$$

\section{Nomenclature}

$C$ : $\quad$ gas concentration $\left(\mathrm{mol} \cdot \mathrm{cm}^{-3}\right)$

$C_{0}$ : total concentration of gas $\left(\mathrm{mol} \cdot \mathrm{cm}^{-3}\right)$

$d_{\text {iron }}$ : apparent density of iron in the reduced pellet $\left(\mathrm{g}\right.$-atom $\left.\cdot \mathrm{cm}^{-3}\right)$

$d_{\text {oxide }}$ : apparent density of iron oxide in the reoxidized pellet $\left(\mathrm{g} \cdot \mathrm{cm}^{-3}\right)$

$D_{e}: \quad$ effective diffusivity $\left(\mathrm{cm}^{2} \cdot \mathrm{sec}^{-1}\right)$

$D_{\mathrm{N}_{2}-\mathrm{O}_{2}}$ : gas diffusivity in the system $\mathrm{N}_{2}-\mathrm{O}_{2}\left(\mathrm{~cm}^{2}\right.$. $\sec ^{-1}$ )

$F: \quad$ reoxidation degree $(\%)$

$G_{1}$ : gas reactant

$J: \quad$ flux of mass transfer $\left(\mathrm{mol} \cdot \mathrm{cm}^{-2} \cdot \mathrm{sec}^{-1}\right)$

$k$ : rate constant of logarithmic rate law (-)

$k_{f}$ : mass transfer coefficient in the gas film $(\mathrm{cm}$. $\left.\sec ^{-1}\right)$

$M_{\mathrm{O}}$ : atomic weight of oxygen

$M_{\mathrm{Fe}}$ : atomic weight of iron

$r$ : radius of unreacted core $(\mathrm{cm})$

$r_{0}: \quad$ radius of pellet $(\mathrm{cm})$

$R$ : ratio of the reoxidation degree at $t$ to the initial reoxidation degree $(-)$

$R_{e}$ : Reynolds number
$S_{1}$ : $\quad$ solid reactant

$S_{2}$ : $\quad$ solid product

Sc: $\quad$ Schmidt number

Sh: Sherwood number

$S_{W}: \quad$ specific surface area $\left(\mathrm{cm}^{2} \cdot \mathrm{g}^{\left.- \text {iron }^{-1}\right)}\right.$

$t: \quad$ reoxidation time $(\mathrm{sec})$

$t_{0}: \quad$ characteristic time (sec)

$v$ : flow rate $\left(\mathrm{cm}^{3} \cdot \mathrm{cm}^{-2} \cdot \mathrm{sec}^{-1}\right)$

$x$ : thickness of oxide layer $(\mathrm{cm})$

$x_{0}$ : characteristic length $(\mathrm{cm})$

$y$ : number of oxygen atoms combined with one atom of iron

$\alpha: \quad$ initial reoxidation degree expressed in the unit $\left(\right.$ mol-oxygen $\cdot g$-atom-iron ${ }^{-1}$ )

$\rho$ : variable showing the distance from the center of pellet $(\mathrm{cm})$

Subscripts and Superscripts

1: reactive gas

2: inert gas

$c$ : bulk flow

$d$ : diffusion

$e$ : equilibrium

expt: experimental value

calc: theoretically calculated value

\section{REFERENCES}

1) S. Kondo, R. Matsumoto and K. Wada: Tetsu-to-Hagané, 50 (1964), 1648.

2) Y. Iguchi and M. Inouye: Tetsu-to-Hagané, 56 (1970), 507.

3) O. Kubaschewski and B. E. Hopkins: Oxidation of Metals and Alloys, (1962), Butterworths and Co. Ltd., London.

4) J. C. Measor and K. K. Afzulpurkar: Phil. Mag., 107 (1964), 817.

5) Y. Iguchi and M. Inouye: Tetsu-to-Hagané, 56 (1970), 671.

6) For example, K. Fueki and S. Kurihara: Bull. Japan Inst. Metals, 8 (1969), 20.

7) M. Ishida and C. Y. Wen: AIChE, 14 (1968), 311.

8) Kagaku Kogaku Kyokai: Handbook of Chem. Eng., (1968), 60, Maruzen Co.

9) W. Jost: Chem. Eng. Sci., 2 (1953), 199. 